\title{
Maximal quantum randomness in Bell tests
}

\author{
Chirag Dhara,, , 用 Giuseppe Prettico, 1 , and A. Acín ${ }^{1,2}$ \\ ${ }^{1}$ ICFO-Institut de Ciencies Fotoniques, Mediterranean Technology Park, 08860 Castelldefels (Barcelona), Spain \\ ${ }^{2}$ ICREA-Institució Catalana de Recerca i Estudis Avançats, Lluis Companys 23, 08010 Barcelona, Spain
}

(Dated: October 29, 2018)

\begin{abstract}
The non-local correlations exhibited when measuring entangled particles can be used to certify the presence of genuine randomness in Bell experiments. While non-locality is necessary for randomness certification, it is unclear when and why non-locality certifies maximal randomness. We provide a simple argument to certify the presence of maximal local and global randomness based on symmetries of a Bell inequality and the existence of a unique quantum probability distribution that maximally violates it. We prove the existence of $\mathrm{N}$-party Bell tests attaining maximal global randomness, that is, where a combination of measurements by each party provides $\mathrm{N}$ perfect random bits.
\end{abstract}

Introduction. Quantum theory radically departs from classical theory in many aspects. Quantum theory, for instance, predicts correlations among distant noncommunicating observers that cannot be reproduced classically. These correlations are termed non-local and violate those conditions known as Bell inequalities that, in contrast, are satisfied by classically correlated systems 11. Quantum theory also incorporates a form of randomness in its framework that does not have a classical counterpart. There is no true randomness in Newtonian physics, as the complete knowledge of initial conditions along with interactions of a system allows one to predict its future dynamics deterministically. As well known however, predictions in quantum systems are necessarily probabilistic. Since violation of Bell inequalities implies that quantum theory cannot be explained by local deterministic theories, the probabilistic nature must arise from intrinsic randomness. Hence, the violation of a Bell inequality certifies the existence of genuine randomness (for recent developments, see [3] and references therein).

The relation between non-locality and randomness has attracted the interest of physicists since the very inception of quantum theory. While earlier motivated by its foundational implications, it has acquired a practical aspect due to the rapid developments in quantum information and computation. Randomness constitutes a valuable information resource, with applications ranging from cryptographic protocols and gambling to numerical simulations of physical and biological systems. Recently, tools to certify and quantify the presence of randomness in Bell tests have been presented in 4. An important advantage of this approach is that it is derived in the deviceindependent scenario, where it is possible to characterize the system from an input-output perspective without regard for its internal working. While, as said, we now have tools to link quantum randomness and non-locality, we are still far from understanding the exact relation between these two quantum properties. For instance, there are situations in which a probability distribution with maximal non-locality does not necessarily contain maximal randomness. Even more counter intuitively, distributions with arbitrarily small non-locality can contain almost maximal randomness in some cases [5]. Along this direction, identifying those quantum set-ups, namely Bell tests, which offer the highest possible randomness would be a highly desirable result, both from a fundamental and practical point of view. This is the main goal of the present work.

It is worth illustrating our motivations with an example. Consider the standard Clauser-Horne-Shimony-Holt (CHSH) inequality [6], $I_{C H S H}=\left\langle A_{1} B_{1}\right\rangle+\left\langle A_{1} B_{2}\right\rangle+$ $\left\langle A_{2} B_{1}\right\rangle-\left\langle A_{2} B_{2}\right\rangle$. At the point of maximal quantum violation, any measurement output by any of the parties provides a perfect random bit. That is, the corresponding probability distribution contains locally the maximum possible of one bit of randomness for every party and every measurement setting. However, there are strictly less than 2 random bits globally, as any pair of local measurements gives correlated results. Now, consider the following modification of the $\mathrm{CHSH}$ inequality, $I_{\eta}=\left\langle A_{1} B_{1}\right\rangle+\left\langle A_{1} B_{2}\right\rangle+\left\langle A_{2} B_{1}\right\rangle-\left\langle A_{2} B_{2}\right\rangle+\eta\left\langle A_{1}\right\rangle$. At the point of maximal quantum violation, only the measurement $A_{2}$ defines a perfect random bit [5]. Why this setting and not the others? Why all of them in the case of CHSH? More in general, when can we expect maximal local, and global randomness in a Bell test?

Our main result is a simple method to infer when and which settings in a Bell test certify perfect random bits. Given a Bell inequality, our method (i) assumes that the quantum probability distribution attaining its maximal violation is unique and (ii) exploits the symmetries of the inequality. We show how this method reproduces all known results relating Bell tests and maximal randomness. Moreover, based on our construction, we provide Bell tests certifying the maximal global randomness in a robust manner, that is, Bell tests for which there exist measurements by the $N$ parties providing $N$ random 
bits. We also provide a geometric interpretation of our findings. Finally, we discuss the existence of uniqueness and show that it is known to exist in several important cases either analytically or from numerical computation.

We start by explaining our notation and stating the basic definitions we use in the text.

Bell tests and quantum distributions. We denote by $(N, M, d)$ a standard Bell experiment consisting of $N$ separated and non-communicating parties, where each of them can perform $M$ local measurements of $d$ outcomes. By repeating the experiment, it is possible to assign a probability distribution $P\left(a_{1}, \ldots, a_{N} \mid x_{1}, \ldots, x_{N}\right)$, where $a_{i}$ is the outcome of a measurement $x_{i}$ by party $1 \leq i \leq N$. We often consider cases with dichotomic measurements i.e. $d=2$. In this case, we can use the following useful parametrization,

$$
P(\mathbf{a} \mid \mathbf{x})=\frac{1}{2^{N}}\left(1+\sum_{i=1}^{N} a_{i}\left\langle A_{i}\right\rangle+\sum_{i<j} a_{i} a_{j}\left\langle A_{i} A_{j}\right\rangle+\sum_{i<j<k} a_{i} a_{j} a_{k}\left\langle A_{i} A_{j} A_{k}\right\rangle+\cdots+a_{1} a_{2} \ldots a_{N}\left\langle A_{1} A_{2} \ldots A_{N}\right\rangle\right)
$$

Here, measurement outputs are labeled by \pm 1 and $\left\langle A_{i} \ldots A_{j}\right\rangle$ are the standard correlators $\left\langle A_{i} \ldots A_{j}\right\rangle=$ $\operatorname{Pr}\left(A_{i} \ldots A_{j}=+1\right)-\operatorname{Pr}\left(A_{i} \ldots A_{j}=-1\right)$.

Randomness. We follow [4,5] and adopt an operational approach where randomness is related to the probability of correctly guessing the outcome of some joint measurement, $\mathbf{x}=\left(x_{1}, x_{2}, \ldots, x_{N}\right)$. We use the guessing probability, $P_{G}(P ; \mathbf{x})=\max _{\mathbf{a}} P(\mathbf{a} \mid \mathbf{x})$, where $\mathbf{a}=\left(a_{1}, a_{2}, \ldots, a_{N}\right)$. The proper measure of intrinsic randomness requires optimizing over all realizations of the observed correlations $G(P ; \mathbf{x})=\max \sum_{i} \lambda_{i} P_{G}\left(P_{i} ; \mathbf{x}\right)$, where the maximization is over all convex decompositions $P(\mathbf{a} \mid \mathbf{x})=\sum_{i} \lambda_{i} P_{i}(\mathbf{a} \mid \mathbf{x})$. It is convenient to express the randomness in bits with the min-entropy, $H_{\infty}(P ; \mathbf{x})=-\log _{2} G(P ; \mathbf{x})$. Note that in a general $(N, M, d)$ scenario there can be at most $\log _{2} d$ bits of local and $N \log _{2} d$ bits of global randomness at any given round of the experiment. For a given $\mathbf{x}=\mathbf{x}_{\mathbf{0}}$, maximal randomness is obtained from a uniform distribution $P\left(\mathbf{a} \mid \mathbf{x}_{\mathbf{0}}\right)=1 / d^{N}, \forall \mathbf{a}$. When $d=2$, this occurs if, and only if, all the correlators appearing in (1) are zero.

Maximal randomness certification. The main result of our work is a simple method to infer when some settings in a Bell test can provide maximal randomness. We assume in what follows that the quantum distribution attaining the maximal quantum violation of the Bell inequality is unique (discussed later). Under this assumption, we show how symmetries in the Bell inequality under permutation of measurement results, possibly together with permutations of measurement settings, lead to maximal randomness. Our method, then, can be summarized as follows: uniqueness plus symmetries implies maximal randomness.

To illustrate our method, it is worth reexamining the examples given above. Consider again the CHSH inequality and denote by $\mathcal{P}^{*}$ the distribution attaining its maximal quantum violation, namely $I_{C H S H}\left(\mathcal{P}^{*}\right)=2 \sqrt{2}$. Note that in this case, this distribution is known to be unique [7]. The symmetry transformation $\mathcal{T}_{s}: a_{1,2} \mapsto$ $-a_{1,2}$ and $b_{1,2} \mapsto-b_{1,2}$ flips the signs of all the onebody correlators, $\left\langle A_{i}\right\rangle$ and $\left\langle B_{j}\right\rangle$, while keeps unchanged all two-body correlators, $\left\langle A_{i} B_{j}\right\rangle$. Applying $\mathcal{T}_{s}$ to $P^{*}$ we obtain a new distribution $\mathcal{T}_{s}\left(\mathcal{P}^{*}\right)=\mathcal{P}^{* *}$ with

$$
\left\langle A_{i}\right\rangle^{* *}=-\left\langle A_{i}\right\rangle^{*}, \quad\left\langle B_{j}\right\rangle^{* *}=-\left\langle B_{j}\right\rangle^{*},
$$

and that also maximally violates $\mathrm{CHSH}$. Because of the uniqueness of the distribution, $\mathcal{P}^{*}=\mathcal{P}^{* *}$ and all onebody correlators (2) must be zero, which certifies 1 bit of local randomness (for both parties). Moving to $I_{\eta}$, the transformation $a_{2} \mapsto-a_{2}, B_{1} \leftrightarrow B_{2}$, flips the value of $\left\langle A_{2}\right\rangle$ without changing the value of $I_{\eta}$. Under the assumption of uniqueness, this proves that the setting $A_{2}$ is fully random. A little thought shows that it is impossible to construct similar transformations for the other local measurements. Our argument, then, easily reproduces the known results for these two inequalities.

As mentioned, our method applies to any Bell inequality with symmetries. The previous argument for the CHSH inequality can be easily generalized to all the chained inequalities of Refs. 8, 9]. Under the assumption of uniqueness, these inequalities always certify 1-dit of local randomness. The chained Bell inequalities can be compactly represented as [9]:

$$
C_{d}^{M}=\sum_{i=1}^{M}\left\langle\left[A_{i}-B_{i}\right]_{d}\right\rangle+\left\langle\left[B_{i}-A_{i+1}\right]_{d}\right\rangle \geqslant d-1
$$

where $A_{i}, B_{j} \in\{0, \ldots, d-1\}$ are measurement choices for Alice and Bob and $A_{M+1}=A_{1}+1$. The square brackets denote sum modulo $d$.

Let $P$ attain the quantum maximum of $C_{d}^{M}$. The transformation $\mathcal{T}: a_{i} \mapsto a_{i}+1$ and $b_{i} \mapsto b_{i}+1$ for every 
$i$ changes the value of the marginal distributions of Alice and Bob but leaves the terms in $C_{d}^{M}$ unchanged. Applying $\mathcal{T}$ to $P$ and assuming it to be unique, it follows that all local distributions of Alice and Bob must be uniform. In other words, the chained inequality certifies $\log _{2} d$ bits of local randomness for every measurement by each party.

Bell tests attaining maximal global randomness. A natural open question is whether there exist Bell tests in the $(N, M, d)$ scenario that allow certifying the maximal possible randomness, namely $N \log _{2} d$ bits. Some progress on this question was obtained in [5], where it was shown how to get arbitrarily close to two random bits in the $(2,2,2)$ scenario. However the corresponding correlations are non-robust. Here, we show how our method can be easily applied to design Bell tests allowing maximal randomness certification in a robust manner.

We start with the bipartite case. Maximal global randomness is impossible in the CHSH case, as at the point of maximal violation all settings are correlated. Maximal global randomness, however, can be certified as soon as another measurement is included. More in general, consider the chained inequalities for an odd number of two-outcome measurements. We move to the notation $a_{i}, b_{j}= \pm 1$ and reexpress (3) as follows:

$$
C_{2}^{M}=\left|\sum_{i=1}^{M}\left\langle A_{i} B_{i}\right\rangle+\sum_{i=1}^{M-1}\left\langle A_{i+1} B_{i}\right\rangle-\left\langle A_{1} B_{M}\right\rangle\right|
$$

where $A_{i}, B_{j}= \pm 1$. Let $M=2 k+1$. As above, we consider a transformation leaving $C_{2}^{M}$ unchanged but under which $\left\langle A_{1} B_{k+1}\right\rangle \mapsto-\left\langle A_{1} B_{k+1}\right\rangle$. Such a transformation is: $\mathcal{T}: a_{1} \mapsto-a_{1}, B_{1+i} \leftrightarrow B_{M-i}, A_{2+i} \leftrightarrow A_{M-i}$ $\forall i 0 \leq i \leq k-1$. Assuming that the distribution maximally violating (4) is unique leads to $\left\langle A_{1} B_{k+1}\right\rangle=0$. The previous results show that $\left\langle A_{1}\right\rangle=0=\left\langle B_{k+1}\right\rangle$. These together certify 2 bits of global randomness for $\left(A_{1}, B_{k+1}\right)$. Similar arguments certify maximal randomness in all inputs of the form $\left(A_{l}, B_{k+l}\right) \forall 1 \leq l \leq k$. Analogous to the case for $\mathrm{CHSH}$, maximal randomness cannot be certified for those measurement combinations appearing in the chained inequality, as they display non-zero correlations. The previous results rely on the assumption of uniqueness, which is unknown for the case of the chained inequality with $M>2$. We then follow [4] and apply the techniques in [10] to get an upper bound on the randomness of $\left(A_{1}, B_{2}\right)$ for the chained inequality with 3 measurement settings. The obtained results corroborate the presence of maximal global randomness, up to numerical accuracy.

We now move to the multipartite case. More precisely, we consider the Mermin inequalities 11 and prove that they allow certifying up to $N$ bits of global randomness for arbitrary odd $N$. Mermin inequalities of $N$ parties are defined recursively as,

$$
M_{N}=\frac{1}{2} M_{N-1}\left(A_{N}+A_{N}^{\prime}\right)+\frac{1}{2} M_{N-1}^{\prime}\left(A_{N}-A_{N}^{\prime}\right)
$$

where $M_{2}$ is the CHSH inequality and $M_{N-1}^{\prime}$ is obtained from $M_{N-1}$ by exchanging all $A_{j}$ and $A_{j}^{\prime}$.

Let $M_{N}$ denote a Mermin inequality of $N=2 J+1$ sites. Party $i$, with $i=1, \ldots, N$ has a choice between two dichotomic measurements, $A_{i}$ and $A_{i}^{\prime}$. It is easily checked that for odd $N, M_{N}$ contains only full correlators with an odd number of primes. We show, using symmetry arguments, that at the point of maximal quantum violation every correlator $\left\langle A_{i} \ldots A_{j}\right\rangle$ (involving an arbitrary number of measurements) that does not appear in $M_{N}$ is identically zero. This automatically implies that any combination of $N$ settings not appearing in the inequality define $N$ random bits.

To see this, first take a specific $N$-body correlator not appearing in $M_{N},\left\langle X_{1} X_{2} \ldots X_{N}\right\rangle$ where $X_{i}=A_{i}$ or $A_{i}^{\prime}$ but such that the total number of primed $A$ is an even number. Denote the outcome of $X_{i}$ by $x_{i}$. Choose any of the parties, say the first one, and denote by $\operatorname{Corr}\left(X_{1}\right)$ the set of all correlators of arbitrary size containing $X_{1}$ plus possibly other settings $X_{i}$ with $i>1$. We would like to show that every element belonging to $\operatorname{Corr}\left(X_{1}\right)$ is equal to zero for the unique distribution maximally violating the inequality. Let us consider the transformation $\mathcal{S}_{1}:\left\{x_{1} \mapsto-x_{1}\right.$, and $x_{j}$ untouched $\forall j>1\}$. This maps $\operatorname{Corr}\left(X_{1}\right) \mapsto-\operatorname{Corr}\left(X_{1}\right)$. The terms in $M_{N}$ remains unchanged if we complement $\mathcal{S}_{1}$ with $\mathcal{S}_{1}^{\prime}:\left\{x_{j}^{\prime} \mapsto-x_{j}^{\prime} \forall j>1\right\}$, where we use $\left(A_{i}^{\prime}\right)^{\prime}=A_{i}$. In fact, note that for the original even primed term we started with, $\mathcal{S}_{1}^{\prime} \circ \mathcal{S}_{1}\left\langle X_{1} X_{2} \ldots X_{N}\right\rangle=-\left\langle X_{1} X_{2} \ldots X_{N}\right\rangle$. The Mermin inequality consists only of odd-parity fullcorrelators. Any such a term can be obtained from $\left\langle X_{1} X_{2} \ldots X_{N}\right\rangle$ by swapping inputs at an odd number of places. However, the transformation $\mathcal{S}_{1}^{\prime} \circ \mathcal{S}_{1}$ is such that at every site, either the outcome of $A_{i}$ or $A_{i}^{\prime}$ flips sign but not both. Hence, $\mathcal{S}_{1}^{\prime} \circ \mathcal{S}_{1}$ applied on any correlator obtained by an odd number of local swaps on $\left\langle X_{1} X_{2} \ldots X_{N}\right\rangle$ gains an additional factor of -1 for each swapped site relative to $\mathcal{S}_{1}^{\prime} \circ \mathcal{S}_{1}\left\langle X_{1} X_{2} \ldots X_{N}\right\rangle$. Thus, $M_{N}$ remains unchanged. It remains to study the effect of $\mathcal{S}_{1}^{\prime}$ on $\operatorname{Corr}\left(X_{1}\right)$. Since $X_{j}^{\prime} \notin \operatorname{Corr}\left(X_{1}\right)$, this set is unmodified under $\mathcal{S}_{1}^{\prime}$, so $\mathcal{S}_{1}^{\prime} \circ \mathcal{S}_{1}$ maps $\operatorname{Corr}\left(X_{1}\right) \mapsto-\operatorname{Corr}\left(X_{1}\right)$. We then conclude from uniqueness that all the correlators in $\operatorname{Corr}\left(X_{1}\right)$ must be zero. The same argument can be run for any party, and then for any full-correlator with an even number of primes, proving the result.

Before concluding this part, it is worth mentioning that similar arguments when applied to the Mermin inequality for even $N$ allow certifying $(N-1)$ bits of randomness.

Geometric interpretation. The previous argument crucially relies on the assumption that there is a unique quantum distribution attaining the maximal violation of a given Bell inequality. For some cases, such as Mermin 
$(N, 2,2)$, this uniqueness has been proven [12, 13] and, then, it is no longer an assumption. For the chained inequality, we have numerical evidence using the techniques from 10 that the distribution saturating it is unique in the $(2,3,2)$ and $(2,4,2)$ cases.

From a geometrical point of view, it is natural to expect that the maximal violation of a generic Bell inequality is attained by a unique point. The set of quantum correlations defines a convex set in the space of probability distributions $P\left(a_{1}, \ldots, a_{N} \mid x_{1}, \ldots, x_{N}\right)$. A Bell inequality is a hyperplane in this space. The maximal quantum violation corresponds to the point in which the hyperplane, i.e. the Bell inequality, becomes tangent to the set of quantum correlations. Since the set is convex, this point is expected to be unique, in general. Of course, there may be situations for which this is not true. So far the only exceptions we have found from numerics are for lifted Bell inequalities. A tight Bell inequality of a smaller space can be lifted in a sense made precise in [14] to a tight Bell inequality in a higher space, either with more parties, measurements or outcomes. For example, $(C H S H-2)_{A B} \otimes C_{1} \leq 0$ is a tight Bell inequality of $(3,2,2)$ in which party $C$ only applies one measurement. It is easy to see that there are several quantum realizations attaining the maximal violation of this inequality. However, it may be argued that these Bell inequalities should be properly be considered as belonging to a lower dimensional space.

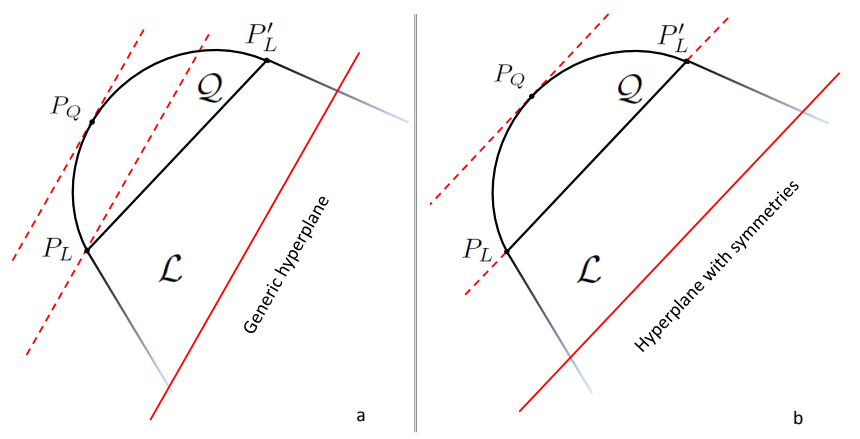

FIG. 1: a) A generic hyperplane generally does not have symmetries and has a unique maximum in both the local and the quantum sets. b) A hyperplane with symmetries (such as the $\mathrm{CHSH}$ ) precludes uniqueness in the local set but still allows for a unique maximum in the quantum set.

One should, however, be careful when following this geometrical intuition. Note that the previous argument does not make use of any quantum property. In fact, the set of classical correlations is also convex and, thus, a generic hyperplane is expected to become tangent at a unique extremal point, see Fig. 11. However, randomness cannot be certified by classical correlations. The reason is that our method applies only to Bell inequalities that are symmetric under permutation of some of the measurement results, possibly assisted by permutations of measurements. It is easy to see that, within the local set, any symmetry under permutations of the results can be immediately used to construct another extremal and deterministic point saturating the inequality as in Fig. 1p.

How do these considerations extend to general nonsignalling correlations? While this is beyond the scope of the present work, we just pointed out here that the chained inequality allows certifying at most one bit of global randomness [15], as opposed to the two bits in the quantum case. This implies that there is more than one non-signalling point saturating the inequality. Understanding why randomness certification, based on uniqueness and symmetries, behaves so differently in the quantum set is an interesting question that deserves further investigation. From a speculative point of view, the fact that the quantum set is not a polytope, as opposed to the set of classical and non-signalling correlations, may play a key role in these considerations.

Conclusions. Our argument is based on the simultaneous existence of uniqueness and symmetries. While in the classical case the needed symmetries immediately break the uniqueness of the maximal violation, this is no longer the case for quantum correlations, as implied by our results. Furthermore, we are yet to find an example where results from our symmetry arguments are in contradiction with numerical results where such computation was possible. For instance, for the I3322 [16 18 inequality, there are no symmetry arguments possible in order to certify maximal local randomness and, in fact, the known maximal quantum violation of the inequality gives non-uniform marginals 19 .

While our simple recipe does not constitute a formal proof of randomness unless uniqueness is proven it still turns out to be very useful to find the right Bell inequalities and measurements allowing maximal randomness certification. Indeed, the results derived following our method can later be confirmed using the techniques from [4, 10. In this sense, we are not aware of any Bell test leading to maximal randomness, local or global, that cannot be explained using our method. Our findings indicate that settings not appearing in the Bell inequality may have more global randomness than those appearing in the inequality. Moreover, using our method, we easily demonstrated the existence of Bell tests allowing maximal global randomness. Finally, our work opens new perspectives on the relation between randomness and nonlocality that deserve further investigation.

We acknowledge support from the ERC Starting Grant PERCENT, the EU Projects Q-Essence and QCS, the Spanish MICIIN through a Juan de la Cierva grant and the Spanish FPI grant, an FI Grant of the Generali- 
tat de Catalunya and projects FIS2010-14830, ExploraIntrinqra, CHIST-ERA DIQIP.

* Electronic address: chirag.dhara@icfo.es

† Electronic address: giuseppe.prettico@icfo.es

[1] J.S. Bell, Physics, 1, 195 (1964).

[2] A. Einstein, B. Podolsky and N. Rosen, Phys. Rev. 47, 777 (1935).

[3] R. Gallego et. al., arXiv:1210.6514

[4] S. Pironio et. al., Nature 464, 1021 (2010).

[5] A. Acín, S. Massar and S. Pironio, Phys. Rev. Lett. 108, 100402 (2012).

[6] J. F. Clauser, M. A. Horne, A. Shimony and R. A. Holt, Phys. Rev. Lett. 23, 880 (1969).

[7] B. S. Tsirel'son, Journal of Soviet Mathematics 36:4, 557
(1987).

[8] S. L. Braunstein and C. M. Caves, Ann. of Phys. 202, 22 (1990).

[9] J. Barrett, A. Kent and S. Pironio, Phys. Rev. Lett. 97, 170409 (2006).

[10] M. Navascués, S. Pironio and A. Acín, Phys. Rev. Lett. 98, 010401 (2007); New J. Phys. 10, 073013 (2008).

[11] N.D. Mermin, Phys. Rev. Lett. 65, 1838-1840 (1990).

[12] R.F. Werner, M.M. Wolf, Phys. Rev. A 64, 032112 (2001).

[13] T. Franz, F. Furrer, R.F. Werner, Phys. Rev. Lett. 106, 250502 (2011); see also arXiv:1010.1131 2.

[14] S. Pironio, J. Math. Phys. 46, 062112 (2005).

[15] N. Jones, L. Masanes, arXiv:quant-ph/0506182 1

[16] M. Froissart, Nuov. Cim. B 64, 241 (1981).

[17] C. Śliwa, Phys. Lett. A 317, 165 (2003).

[18] D. Collins and N. Gisin, J. Phys. A: Math. Gen. 37, 1775 (2004).

[19] T. Vertesi, private communication. 